\title{
Immunoglobulin G4-Related Lung Disease Presenting as Lung Cavitating Mass and Mimicking Lung Cancer
}

\author{
Liang-jun XIE, ${ }^{1}$ Jian-fang LI, ${ }^{1}$ Zhi LIU, ${ }^{2}$ Feng ZHANG, ${ }^{1}$ Chang ZHAO, ${ }^{3}$ \\ Lu-ping QIN, ${ }^{1}$ Ting-jie ZHANG, ${ }^{1}$ Mu-hua $\mathrm{CHENG}^{1}$ \\ ${ }^{1}$ Department of Nuclear Medicine, The Third Affiliated Hospital of Sun Yat-sen University, Guangzhou, China \\ ${ }^{2}$ Department of Ultrasound, Nanfang Hospital of Southern Medicial University, Guangzhou, China \\ ${ }^{3}$ Department Pathology, The Third Affiliated Hospital of Sun Yat-sen University, Guangzhou, China
}

\begin{abstract}
Immunoglobulin G4-related lung disease (IgG4-RLD) is a disease in which abundant activated IgG4-positive plasma cells and lymphocytes infiltrate lung tissues with high ${ }^{18} \mathrm{~F}$-fluorodeoxyglucose uptake. Although various forms of radiologic features of IgG4-RLD have been reported, cavitating mass is a rare imaging feature and should be differentiated from cancer. Therefore, in this article, we report two cases both with unprovoked cough, bloody sputum and presenting quite similar cavitating lesions with high ${ }^{18} \mathrm{~F}$-fluorodeoxyglucose uptake on positron emission tomography/ computed tomography, one of which diagnosed as IgG4-RLD and the other as lung cancer based on biopsy eventually. The awareness of the imaging features of IgG4-RLD and lung cancer described in the present study may help physicians to distinguish one from the other. IgG4-RLD should be considered in the differential diagnosis of cavitary lung lesions.

Keywords: Cavity; ${ }^{18} \mathrm{~F}$-fluorodeoxyglucose; immunoglobulin G4-related lung disease; lung cancer.
\end{abstract}

Immunoglobulin G4-related lung disease (IgG4RLD) is a disease in which IgG4-positive plasma cells and lymphocytes infiltrate lung tissues along with immunohistochemically evident fibrous interstitial proliferation in the background. ${ }^{1,2}$ Since the first report of a patient with IgG4-RLD in $2004,{ }^{3}$ many similar patients have been described. ${ }^{2}$ However, there are scarce cases focusing on IgG4RLD in ${ }^{18}$ F-fluorodeoxyglucose (FDG) positron emission tomography/computed tomography (PET/CT) imaging feature. ${ }^{1,4}$ In addition, the manifestation of IgG4-RLD were mostly described as parenchymal nodules or masses, interstitial lung disease, mediastinal lymphadenopathy, and fibrosing mediastinitis; ;,2,4,5 however, few studies reported cavitating IgG4-RLD. ${ }^{6}$ In this article, we report two cases presenting quite similar cavitating lesions with high ${ }^{18} \mathrm{~F}-\mathrm{FDG}$ uptake on $\mathrm{PET} / \mathrm{CT}$, one of which diagnosed as IgG4-RLD and the other as lung cancer based on biopsy.

\section{CASE REPORT}

Case 1- A 65-year-old nonsmoking Chinese female patient had unprovoked cough accompanied with bloody sputum for one month. However, the patient had no other symptoms such as fever, thorax suffocation or breathing hard. The laboratory reports showed elevated serum level of C-reactive protein $(11.6 \mathrm{mg} / \mathrm{L}$, reference range $0.6-6.0 \mathrm{mg} / \mathrm{L})$, erythrocyte sedimentation rate $(66.00 \mathrm{~mm} / \mathrm{H}$, reference range $0-20 \mathrm{~mm} /$ hour) and depressed hemoglobin 


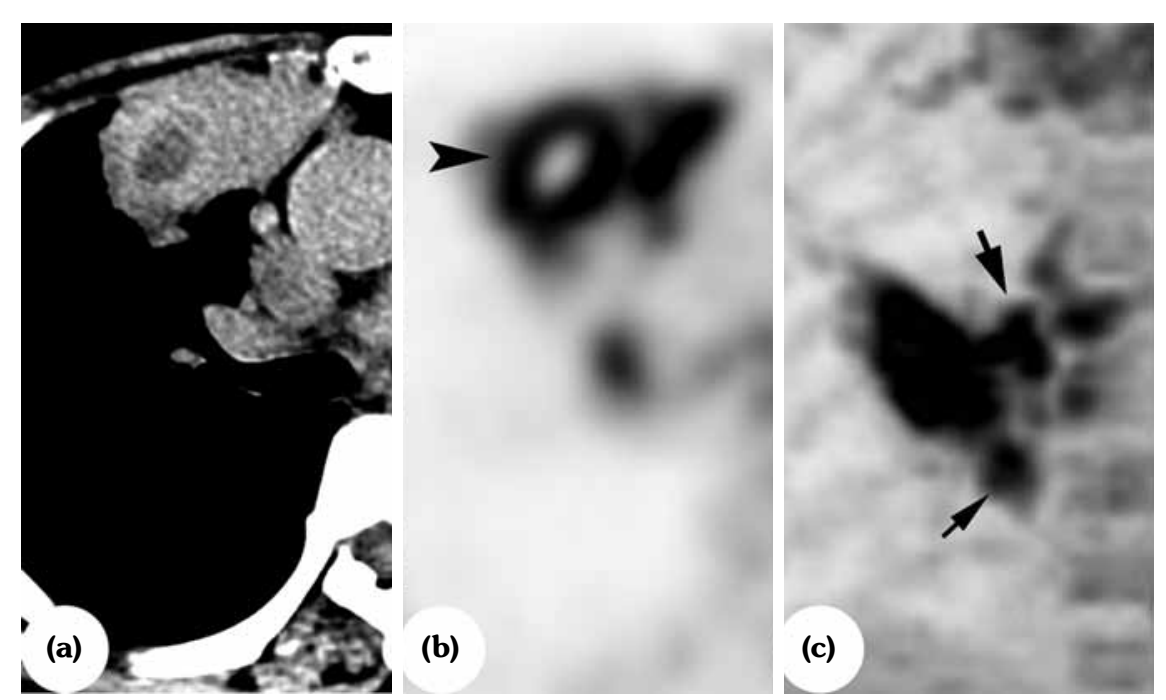

Figure 1. (a) Positron emission tomography/computed tomography imaging displayed a poor-defined $55.9 \times 29.5 \mathrm{~mm}$ irregular lesion with round low-density inside in right S3 segment. (b) Lesion was with a well-demarcated high ${ }^{18}$ F-fluorodeoxyglucose uptake thick-walled cavity measuring $28 \times 23 \mathrm{~mm}$, with a maximum standardized uptake value of 9.7 (arrow head), and (c) right lung hilus and mediastinal lymph node, with a maximum standardized uptake value of 6.45 (arrows).

(112 g/L, reference range 115-150 g/L), while other measured laboratory data were within normal limits including serum leukocyte, tumor markers and anti-mycobacterium tuberculosis antibody. The serum IgG4 was not analyzed initially. A written informed consent was obtained from the patient.

${ }^{18}$ F-fluorodeoxyglucose PET/CT (GE Discovery 690, GE Healthcare, Milwaukee, USA) imaging displayed a poor-defined $55.9 \times 29.5 \mathrm{~mm}$ irregular lesion with round low-density inside in the right S3 segment (Figure 1a) at mediastinal window setting. The interface between the lesion and surrounding organs was unclear but the inner wall was smooth. The lesion was with a well-demarcated high ${ }^{18}$ F-FDG uptake thick-walled cavity measuring $28 \times 23 \mathrm{~mm}$, with a maximum standardized uptake value $\left(\mathrm{SUV}_{\max }\right)$ of 9.7 (Figure $\left.1 \mathrm{~b}\right)$, and the right lung hilus and mediastinal lymph node were enlarged with a SUV $\mathrm{Sax}_{\max }$ of 6.4 (Figure 1c). Lung cancer was initially suspected based on PET/CT finding. However, histopathological examination (ultrasound-guided fine-needle biopsy, Figure 2) demonstrated IgG4-RLD. The serum level of IgG4 was complementarily analyzed and noted to have been slightly elevated to $148 \mathrm{mg} / \mathrm{dL}$ (reference range $\leq 135 \mathrm{mg} / \mathrm{dL}$ ).
Case 2- A 73-year-old smoking Chinese male patient presented with unprovoked cough accompanied with bloody sputum for three months. The laboratory reports showed elevated serum level of erythrocyte sedimentation rate $(25.00 \mathrm{~mm} /$ hour$)$, neuron-specific enolase (NSE, $17.73 \mathrm{ng} / \mathrm{mL}$, reference range 0-16.3 ng/mL), cytokeratin 19 fragment (CYFRA $21-1,8.87 \mathrm{ng} / \mathrm{mL}$, reference range $0-3.3 \mathrm{ng} / \mathrm{mL}$ ), while other measured laboratory data were within normal limits, including serum leukocyte and antimycobacterium tuberculosis antibody. A written informed consent was obtained from the patient.

${ }^{18}$ F-fluorodeoxyglucose PET/CT imaging displayed an ill-defined $40 \times 45 \mathrm{~mm}$ irregular lesion with low-density inside in the right S3 segment (Figure 3a). The interface between the lesion and surrounding organs was unclear and the inner wall was irregular. The lesion was with a circular-nodular high ${ }^{18}$ F-FDG uptake thick-walled cavity measuring $40 \times 36 \mathrm{~mm}$, with $\mathrm{SUV}_{\max }$ of 20.7 (Figure 3b), and the right lung hilus and mediastinal lymph node were enlarged with $\mathrm{SUV}_{\max }$ of 4.7 (Figure 3c). Moderately differentiated lung squamous cell carcinoma was confirmed by histopathological examination (CT-guided fine-needle biopsy, Figure 4). 

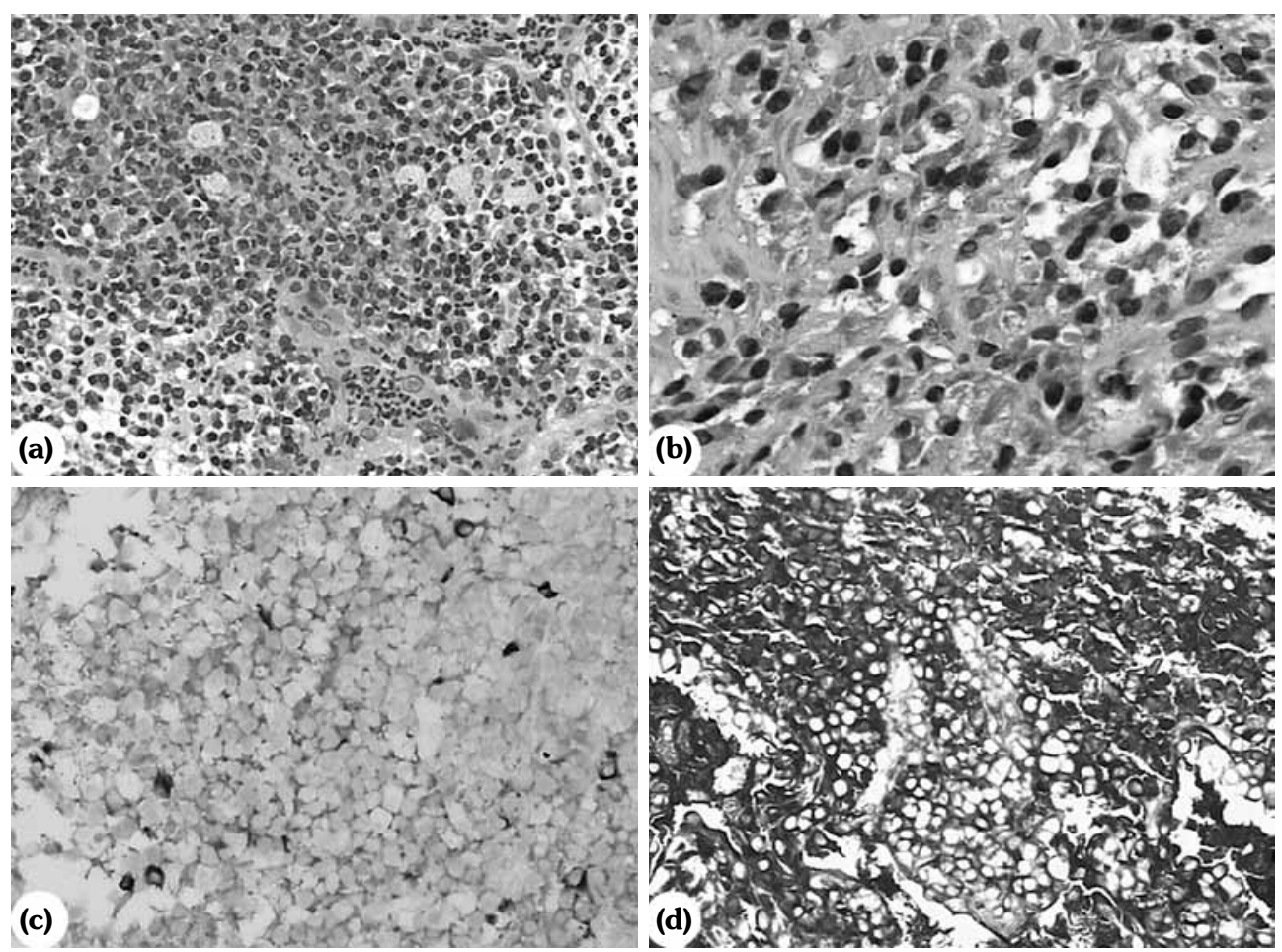

Figure 2. Histopathological examination of Case 1 showed that inflammatory cell infiltration consisting largely of lymphocytes and plasma cells on a background comprised of fibrous interstices with fibrosis, fibroblast proliferation, storiform fibrosis and obliterative phlebitis [a (200x)-b (400x)]. Slides stained for immunoglobulin G4 showed 100\% immunoglobulin G4-positive cells per high-power field and immunoglobulin G4/immunoglobulin G ratio was 50.2-74.6\% [c $(200 \times)$-d $(400 \times)]$.

\section{DISCUSSION}

Radiologic findings of IgG4-RLD can be categorized into four major features: (i) solid nodular densities; (ii) round-shaped groundglass opacities; (iii) alveolar interstitial type with bronchiectasis resembling interstitial lung disease and pulmonary fibrosis with honeycombing; and (iv) thickening of the bronchovascular bundles and interlobular septa. ${ }^{7}$ Because of abundant activated inflammatory cell infiltration, including lymphocytes and plasma cells which utilize anaerobic glycolysis as a source of energy. ${ }^{8}$ the lesions of IgG4-RLD always present high ${ }^{18} \mathrm{~F}-\mathrm{FDG}$ uptake with $\mathrm{SUV}_{\max }$ range 3.4-14.1,4,9,10 In case 1, $\mathrm{PET} / \mathrm{CT}$ revealed an irregular lesion with discrete margins and well-demarcated high ${ }^{18} \mathrm{~F}-\mathrm{FDG}$ uptake thick-walled cavity inside accompanied with enlarged right lung hilus and mediastinal lymph node. This imaging feature was rarely reported in the previous studies.
Taira et al. ${ }^{11}$ reported a case with cavitating lesion in which a lung abscess was initially suspected, but the pleomorphic carcinoma of lung was ultimately confirmed. They suggested that both abscesses and malignancy should be suspected in cases involving masses presenting as ring-enhancing lesion on contrast-enhanced CT. Lung cancer appears as a quite irregular internal and outer contour cavity on CT. ${ }^{12}$ Both of our cases were initially suspected as lung cancer based on the high ${ }^{18} \mathrm{~F}-\mathrm{FDG}$ uptake thick-walled cavitating featured with irregular parenchyma around the cavity and enlarged lymph node. However, Case 1 was with a well-demarcated high ${ }^{18}$ F-FDG uptake, while Case 2 was with a circular-nodular high ${ }^{18}$ F-FDG uptake. Nodular high ${ }^{18} \mathrm{~F}-\mathrm{FDG}$ uptake like nodular enhancement seen in contrast-enhanced CT may be one of the most important features in making a differential diagnosis between intracavitary IgG4-RLD and a cavitating lung cancer. ${ }^{13}$ 

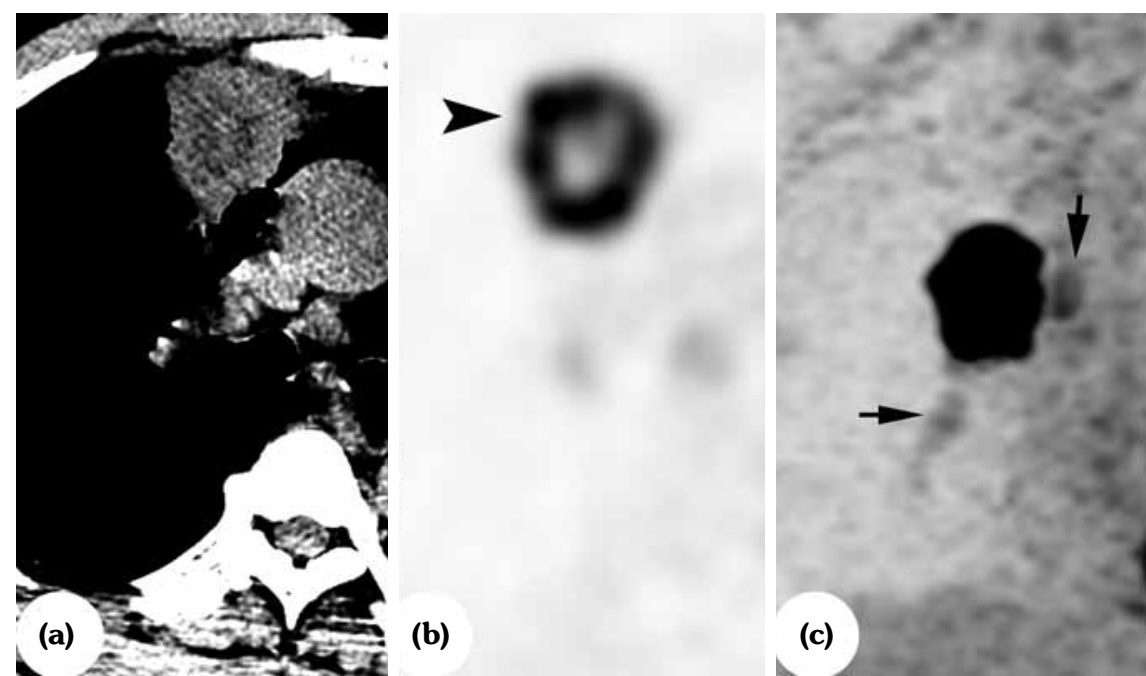

Figure 3. (a) Positron emission tomography/computed tomography imaging displayed a poor-defined $40 \times 45 \mathrm{~mm}$ irregular lesion with irregular low-density inside in right $\mathrm{S} 3$ segment. (b) Lesion was with a circular-nodular high ${ }^{18} \mathrm{~F}$-fluorodeoxyglucose uptake thick-walled cavity measuring $40 \times 36 \mathrm{~mm}$, with maximum standardized uptake value of 20.7 (arrow head), and (c) right lung hilus and mediastinal lymph node were enlarged with maximum standardized uptake value of 4.7 (arrows).

Blood examination should be performed, which play an important role in diagnosing IgG4RLD and lung cancer. The serum level of IgG4 of Case 1 was complementarily analyzed and found to be mildly elevated as most of previous reports. ${ }^{1,2,4,6,10,14}$ Serum level of IgG and IgG4 is the most sensitive and specific laboratory test for the diagnosis of IgG4-RLD. NSE and

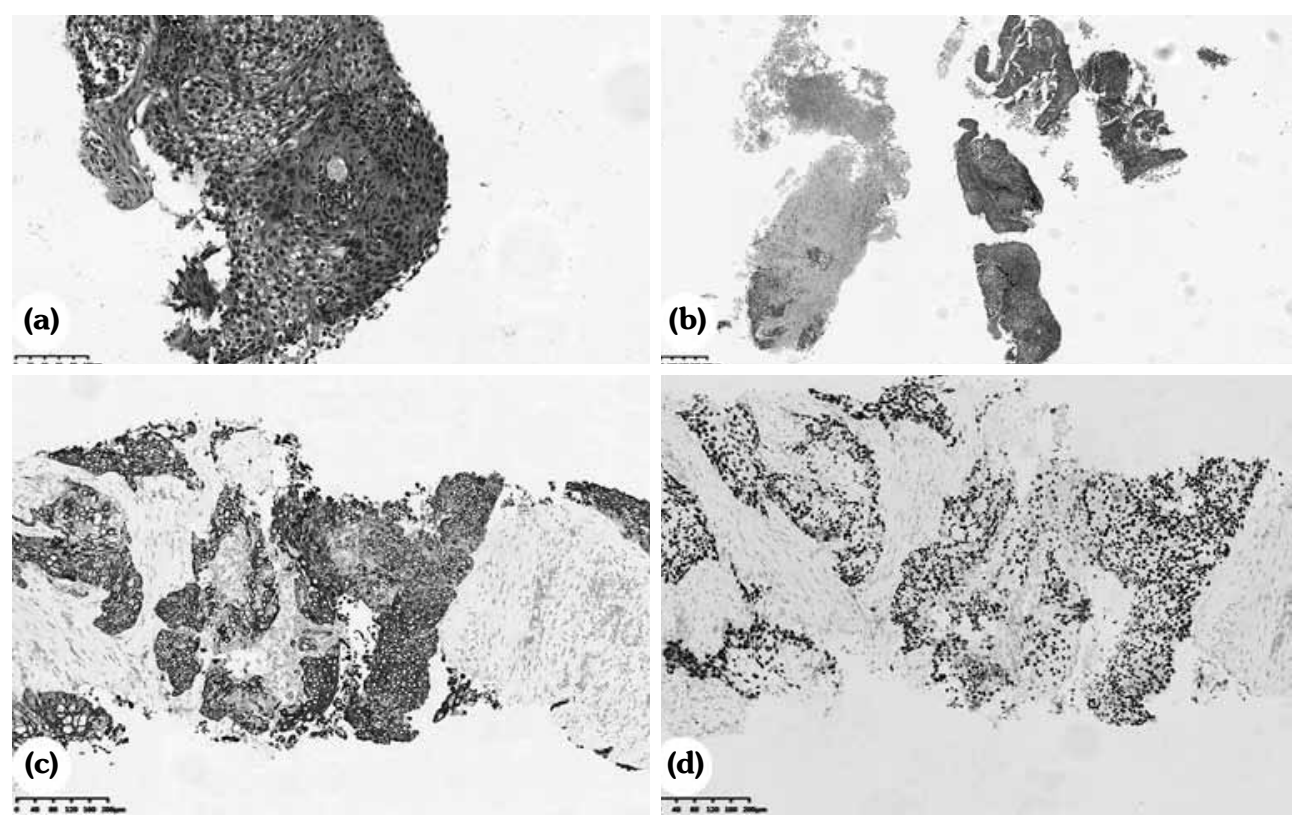

Figure 4. Histopathological examination of Case 2 showed that fibrous tissue was infiltrated by heterotypic cells with nest-like distribution. Cells have big nuclei with hyperchromatism and

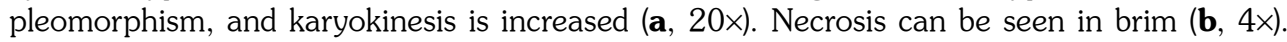
Immunohistochemistry showed cytokeratin 5/6 (+) (c, 10x), P63 (+) (d, 10x), cytokeratin $7(-)$, TTF-1 (-) and Ki67 (50\%). 
CYFRA 21-1 are both tumor markers for primary lung cancer and may be useful complements to standard clinical imaging methods. ${ }^{15}$ The serum level of NSE and CYFRA 21-1 of Case 2 were elevated. However, the cases of Taira et al. ${ }^{11}$ were within normal tumor markers as Case 1. Thus, negative serum NSE and CYFRA 21-1 cannot be used as exclusion criteria of lung cancer.

In conclusion, IgG4-RLD presents various forms of radiologic features and high ${ }^{18} \mathrm{~F}-\mathrm{FDG}$ uptake for abundant activated inflammatory cell infiltration. Cavitary mass is a rare imaging feature of IgG4-RLD and should be differentiated from lung cancer, especially in patients with bloody sputum. Elevated serum IgG4 and nodular high ${ }^{18} \mathrm{~F}-\mathrm{FDG}$ uptake may be one of the most important features in making a differential diagnosis between intracavitary IgG4-RLD and lung cancer.

\section{Declaration of conflicting interests}

The authors declared no conflicts of interest with respect to the authorship and/or publication of this article.

\section{Funding}

The authors received no financial support for the research and/or authorship of this article.

\section{REFERENCES}

1. Kitada M, Matuda Y, Hayashi S, Ishibashi K, Oikawa $\mathrm{K}$, Miyokawa $\mathrm{N}$, et al. IgG4-related lung disease showing high standardized uptake values on FDGPET: report of two cases. J Cardiothorac Surg 2013;8:160.

2. Matsui S, Hebisawa A, Sakai F, Yamamoto H, Terasaki Y, Kurihara Y, et al. Immunoglobulin G4-related lung disease: clinicoradiological and pathological features. Respirology 2013;18:480-7.

3. Taniguchi $T$, Ko M, Seko S, Nishida O, Inoue F, Kobayashi $\mathrm{H}$, et al. Interstitial pneumonia associated with autoimmune pancreatitis. Gut 2004;53:770.

4. Odaka M, Mori S, Asano H, Yamashita M, Kamiya N,
Morikawa T. Thoracoscopic resection for a pulmonary nodule with the infiltrate of IgG4-positive plasma cells. Asian J Endosc Surg 2012;5:176-8.

5. Ryu JH, Sekiguchi H, Yi ES. Pulmonary manifestations of immunoglobulin G4-related sclerosing disease. Eur Respir J 2012;39:180-6.

6. Jinnur PK, Yi ES, Ryu JH, Iyer VN. Cavitating Lung Disease: A Novel Presentation of IgG4-Related Disease. Am J Case Rep 2015;16:478-82.

7. Inoue $\mathrm{D}$, Zen $\mathrm{Y}$, Abo $\mathrm{H}$, Gabata $\mathrm{T}$, Demachi $\mathrm{H}$, Kobayashi $\mathrm{T}$, et al. Immunoglobulin G4-related lung disease: CT findings with pathologic correlations. Radiology 2009;251:260-70.

8. Vaidyanathan S, Patel CN, Scarsbrook AF, Chowdhury FU. FDG PET/CT in infection and inflammation-current and emerging clinical applications. Clin Radiol 2015;70:787-800.

9. Zhang J, Chen H, Ma Y, Xiao Y, Niu N, Lin W, et al. Characterizing IgG4-related disease with (1)(8)F-FDG PET/CT: a prospective cohort study. Eur J Nucl Med Mol Imaging 2014;41:1624-34.

10. Matsumiya R, Hosono O, Yoshikawa N, Uehara M, Kobayashi $\mathrm{H}$, Oda A, et al. Elevated Serum IgG4 Complicated by Pericardial Involvement with a Patchy (18)F-FDG Uptake in PET/CT: Atypical Presentation of IgG4-related Disease. Intern Med 2015;54:2337-41.

11. Taira N, Kawabata T, Gabe A, Ichi T, Kushi K, Yohena $\mathrm{T}$, et al. Lung cancer mimicking lung abscess formation on CT images. Am J Case Rep 2014;15:243-5.

12. Pentheroudakis G, Kostadima L, Fountzilas G, Kalogera-Fountzila A, Klouvas G, Kalofonos C, et al. Cavitating squamous cell lung carcinoma-distinct entity or not? Analysis of radiologic, histologic, and clinical features. Lung Cancer 2004;45:349-55.

13. Park Y, Kim TS, Yi CA, Cho EY, Kim H, Choi YS. Pulmonary cavitary mass containing a mural nodule: differential diagnosis between intracavitary aspergilloma and cavitating lung cancer on contrastenhanced computed tomography. Clin Radiol 2007;62:227-32.

14. Vasaitis L. IgG4-related disease: A relatively new concept for clinicians. Eur J Intern Med 2016;27:1-9.

15. Seemann MD, Beinert T, Fürst H, Fink U. An evaluation of the tumour markers ... (CEA), cytokeratin marker (CYFRA 21-1) and neuron-specific enolase (NSE) in the differentiation of malignant from benign solitary pulmonary lesions. Lung Cancer 1999;26:149-55. 